\title{
Distribución potencial de Pinus cembroides, Pinus nelsonii y Pinus culminicola en el Noreste de México
}

\section{Potential distribution of Pinus cembroides, Pinus nelsonii and Pinus culminicola in northeastern Mexico}

\author{
Mario Alberto García-Aranda ${ }^{1 *}$, Jorge Méndez-González ${ }^{2}$, José Yunior Hernández-Arizmendi ${ }^{3}$ \\ ${ }^{1}$ Especies, Sociedad y Hábitat A.C., Dalí 413 Col. Misión Real. CP. 66640. Apodaca, Nuevo León, México. \\ ${ }^{2}$ Departamento Forestal, Universidad Autónoma Agraria Antonio Narro. Calzada Antonio Narro 1923. Buenavista Saltillo, CP. \\ 25315. Coahuila, México. \\ ${ }^{3}$ Becario de CONACyT, posgrado en Ingeniería en Sistemas de Producción, Universidad Autónoma Agraria Antonio Narro. \\ Calzada Antonio Narro 1923. Buenavista Saltillo, CP. 25315. Coahuila, México. \\ *Autor de correspondencia: mgarcia_20@yahoo.com.mx
}

Artículo científico recibido: 21 de marzo de 2017 aceptado: 12 de agosto de 2017

RESUMEN. El clima del mundo está cambiando de forma significativa en los últimos años y con ello la distribución de las especies. El objetivo del estudio fue generar modelos de distribución potencial de las especies de pinos piñoneros Pinus cembroides, P. culminicola y $P$. nelsonii del noroeste de México. Para establecer el modelo más preciso, se usaron dos escenarios: a) con 19 variables bioclimáticas Worldclim, y b) con las mismas variables más las variables topográficas de altitud, pendiente y exposición. Se modeló con registros REMIB CONABIO, un total de 208, 89 y 67 registros para cada especie con ajuste logístico y 500 iteraciones. Se realizó la prueba de Jacknife para establecer el porcentaje de participación de las variables en el modelo. Los resultados muestran que ambos escenarios predicen de forma adecuada la distribución potencial de las especies estudiadas. De acuerdo al valor AUC, los ajustes del modelo son mejores para las especies de distribución restringida con 0.999 para $P$. nelsonii y $P$. culminicola y de 0.998 para $P$. cembroides, integrando el modelo la altitud, la temperatura mínima y la pendiente, con contribución promedio de 18.6, 16.6 y $32.8 \%$ cada variable. La distribución potencial de $P$. cembroides puede ampliarse en el noreste de México, debido a que el modelo integró de forma significativa variables de temperatura máxima y discriminó aquellas derivadas de la precipitación.

Palabras clave: Nicho ecológico, MaxEnt, piñoneros, Worldclim

ABSTRACT. The world's climate has changed significantly in recent years and with it the distribution of species. The aim of the study was to generate potential distribution models of three pinyon pine species, Pinus cembroides, $P$. culminicola and $P$. nelsonii, of northwestern Mexico. To establish the most accurate model, two scenarios were used: a) with 19 Worldclim bioclimatic variables, and b) with the same variables plus the topographic variables of elevation, slope and exposure. It was modeled with REMIB CONABIO records, a total of 208, 89 and 67 records for each species with logistic adjustment and 500 iterations. The Jacknife test was performed to establish the participation percentage of the variables in the model. The results show that both scenarios adequately predict the potential distribution of the species studied. According to the AUC value, the model fits are better for restricted-distribution species with 0.999 for $P$. nelsonii and P. culminicola and 0.998 for $P$. cembroides, the model integrating elevation, minimum temperature and slope, with an average contribution of $18.6,16.6$ and $32.8 \%$ for each variable. The potential distribution of $P$. cembroides can be extended in northeastern Mexico, because the model significantly integrated maximum temperature variables and discriminated those derived from precipitation.

Key words: Ecological niche, MaxEnt, pinyon pines, Worldclim 


\section{INTRODUCCIÓN}

Los modelos de distribución de especies han evolucionado, el primero fue un programa sencillo como el BIOCLIM (Nix 1986), el modelo utiliza variables climáticas y detecta condiciones ambientales adecuadas para las especies. Después surgieron los modelos de regresión logística y lineal como GLM (Generalised Linear Models) (Austin y Smith 1989). Debido a los problemas derivados del cambio climático, deforestación, pérdida de biodiversidad, entre otros que se presentan en las áreas naturales del mundo, surge la necesidad de implementar nuevas herramientas y tecnologías para la modelación de hábitat de especies forestales, por lo que los modelos BIOCLIM, GLM, GARP (Genetic Algorithm for Rule-set Prediction) y GAP (Groups Algorithms Programming) se llevaron a la inteligencia artificial (Stockwell 1999). La combinación de estos modelos y el desarrollo de los sistemas de información geográfica (SIG), generaron nuevos métodos para la modelación geográfica, como MaxEnt, procedimiento de modelación de nicho ecológico que requieren de variables ambientales y registros de presencia de la especie, que se usan para producir modelos o mapas de distribución potencial de especies (Graham et al. 2004).

Hasta finales de la década pasada, se tenían aproximadamente 15 métodos para la modelación del hábitat de las especies (Martínez 2010). Siendo MaxEnt uno de los métodos de mayor precisión (Kumar y Stohlgren 2009), el cual esta basado en la máxima entropía donde se tienen datos de presencia de especies (Phillips et al. 2006). El método MaxEnt se ha aplicado en la ecología (Valdés et al. 2012), conservación de la biodiversidad (García et al. 2012, Ávila et al. 2014), especies en riesgo de extinción (Kumar y Stohlgren 2009, Aguirre y Duivenvoorden 2010), en la evaluación del impacto del cambio climático sobre la distribución de organismos (Lawler et al. 2009) y en la fauna silvestre (Ortíz et al. 2012, De la Torre y TorresKnoop 2014). Tambíen se ha aplicado en el área forestal para la detección temprana de enfermedades (Meentemeyer et al. 2008), para la búsqueda de zonas afectadas por plagas forestales (Salinas et al. 2010) y para detectar nuevas áreas semilleras de especies amenazadas (Shaily et al. 2010).

Las especies de pinos piñoneros $P$. cembroides, $P$. culminicola y $P$. nelsonii, están adaptadas al clima y la topografía de la sierra madre oriental, $P$. cembroides presenta amplia distribución en México y es fuente importante de ingresos económicos para los pobladores locales. Mientras que $P$. nelsonii y $P$. culminicola tienen distribución restringida (Farjon y Styles 1997, Estrada et al. 2014) con estatus de protección y amenazadas de acuerdo con la NOM059, por lo que la generación de información con modelos de distribución potencial es importante para establecer planes de manejo, para las especies con estatus de conservación (Austin y Meyers 1996). Por lo anterior el objetivo fue generar modelos de distribución potencial para $P$. cembroides, $P$. culminicola y $P$. nelsonii del noreste de México por medio de las variables ambientales.

\section{MATERIALES Y MÉTODOS}

\section{Integración de registros geográficos de las es- pecies}

La fuente de registros de presencia de las especies fue de la Red Mundial de Información sobre la Biodiversidad REMIB (CONABIO 2015) de donde se descargaron los registros (coordenadas geográficas) de presencia de las especies, los cuales se validaron con la distribución conocida de la especie (Perry 1991, Farjon y Styles 1997, Estrada et al. 2014), eliminando registros con coordenadas erróneas, por lo que se obtuvieron 208 registros de $P$. cembroides, 89 de $P$. culminicola y 67 de $P$. nelsonii.

\section{Obtención y edición de cubiertas SIG de variables ambientales}

Del sitio web de WorldClim se obtuvieron cubiertas geográficas de 19 variables ambientales denominadas Bioclim (Tabla 1), éstas representan las históricas anuales de 1950 al 2000 de temperatura y precipitación (Hijmans et al. 2005). Son cubiertas de tipo raster en formato grid genérico compuestas por pixeles con resolución de 30 segundos de 
Tabla 1. Variables climáticas BIOCLIM y topográficas utilizadas para la modelación MaxEnt de la distribución potencial de $P$. cembroides, P. nelsonii y P. culminicola en el Noreste de México.

\begin{tabular}{|c|c|c|c|}
\hline Variable & Descripción (unidad) & Variable & Descripción (unidad) \\
\hline Bio01 & Temperatura media anual $\left({ }^{\circ} \mathrm{C}\right)$ & Bio12 & Precipitación anual (mm) \\
\hline Bio02 & Intervalo medio diurno (temp. max-temp. min) & Bio13 & Precipitación del mes más húmedo (mm) \\
\hline Bio03 & Isotermalidad (Bio02/Bio07) (*100) & Bio14 & Precipitación del mes más seco $(\mathrm{mm})$ \\
\hline Bio04 & $\begin{array}{l}\text { Temporalidad de temperatura (desviación estándar } \\
* 100 \text { ) }\end{array}$ & Bio15 & $\begin{array}{l}\text { Temporalidad de la precipitación (coeficiente de } \\
\text { variación) }\end{array}$ \\
\hline Bio05 & Temperatura máxima del mes más cálido $\left({ }^{\circ} \mathrm{C}\right)$ & Bio16 & Precipitación del $\frac{1}{4}$ anual más húmedo (mm) \\
\hline Bio06 & Temperatura mínima del mes más frio $\left({ }^{\circ} \mathrm{C}\right) \mid$ & Bio17 & Precipitación del $\frac{1}{4}$ anual más seco $(\mathrm{mm})$ \\
\hline Bio07 & Intervalo de temperatura anual (Bio05-Bio06) & Bio18 & Precipitación del $\frac{1}{4}$ anual más cálido (mm) \\
\hline Bio08 & Temperatura media del $\frac{1}{4}$ anual más húmedo $\left({ }^{\circ} \mathrm{C}\right)$ & Bio19 & Precipitación del $\frac{1}{4}$ anual más frio (mm) \\
\hline Bio09 & Temperatura media del $\frac{1}{4}$ anual más seco $\left({ }^{\circ} \mathrm{C}\right)$ & Altitud & Elevación sobre el nivel medio del mar (msnm) \\
\hline Bio10 & Temperatura media del & Exposición & Dirección de facetas de pendiente ( $0-360$ grados) \\
\hline Bio11 & Temperatura media del & Pendiente & Grados de inclinación del terreno (\%) \\
\hline
\end{tabular}

arco ( $1 \mathrm{~km})$, por lo que primero se recortaron para la región noreste de México. Después con el programa ArcMap 10.2 se transformaron a formato grid ASCII. De la página web WorldClim se extrajo la cubierta raster global de altitud, que se usó para obtener la pendiente y la exposición en grados.

\section{Modelación de la distribución potencial de las especies}

La distribución potencial de las tres especies se modeló bajo los siguientes escenarios: a) con el set de 19 variables bioclimáticas y b) con 19 variables bioclimáticas más tres variables topográficas. En todos los casos se empleó el modelo logístico con un máximo de 500 iteraciones, creando curvas de respuesta y pruebas Jacknife para validar los modelos con el $50 \%$ de registros para pruebas de entrenamiento y el resto para las pruebas de modelación. El modelo resultante consiste en una cubierta geográfica formato grid con valores probabilísticos de 0 a 1 de nicho o hábitat potencial, donde 0 indica pixeles que no representan hábitat y valores cercanos a 1 representan mayor probabilidad de ocurrencia de la especie (Phillips et al. 2006).

En la modelación se consideraron pruebas de precisión a través de un análisis de omisión y comisión, una prueba de sensibilidad gráfica de área bajo la curva (AUC) y pruebas de Jacknife que analizan el comportamiento independiente de cada variable bioclimática para determinar su peso y grado de participación en la creación del modelo. Los valores de AUC generados de ambos escenarios se evaluaron entre sí para determinar el mejor modelo para la especie, donde los valores más altos de AUC indican un mejor modelo. De las variables que conformaron el modelo, se obtuvieron sus valores y se realizaron histogramas de frecuencia para conocer los rangos o límites de tolerancia de la especie (García et al. 2012).

\section{Cálculo de superficie del hábitat potencial}

De los valores de probabilidad $(0-1)$ del mejor modelo de cada especie y usando el reclasificador de ArcMap 10.2 se ordenaron los valores en intervalos de 0.1 a partir de los cuales se calculó su superficie por decil para conocer el área potencial correspondiente.

\section{RESULTADOS}

\section{Modelación de las especies con MaxEnt}

En la Tabla 2 se muestran los valores de AUC de ambos escenarios planteados para la modelación MaxEnt para $P$. cembroides, $P$. culminicola y $P$. nelsonii en el noreste de México. El ajuste de los modelos en ambos escenarios es bueno; el valor de AUC encontrado en estas especies es de los más altos reportados en la literatura. La especie $P$. cembroides tiene amplia distribución con valor menor (0.998) en relación al AUC de las otras dos especies que son de distribución restringida (0.999). La Figura 1 muestra el modelo resultante de $P$. cembroides, donde se observa la amplia superficie de distribución potencial (0.8 - 0.85). En la Figura 
Tabla 2. Valores AUC generados para denotar el ajuste del modelo de predicción a través de MaxEnt en $P$. cembroides, $P$. nelsonii y $P$. culminicola en el Noreste de México.

\begin{tabular}{lcccccc}
\hline & \multicolumn{3}{c}{ Especie } \\
\hline & \multicolumn{2}{c}{$\boldsymbol{P .}$ cembroides } & & $\boldsymbol{P}$. nelsonii & \multicolumn{2}{c}{$\boldsymbol{P . ~ c u l m i n i c o l a}$} \\
\cline { 2 - 7 } & Bioclim & Bioclim + topografía & Bioclim & Bioclim + topografía & Bioclim & Bioclim + topografía \\
\hline AUC de entrenamiento & 0.975 & 0.991 & 0.999 & 0.999 & 0.999 & 0.999 \\
AUC de prueba & 0.971 & 0.998 & 0.998 & 0.999 & 0.999 & 0.999 \\
\hline
\end{tabular}

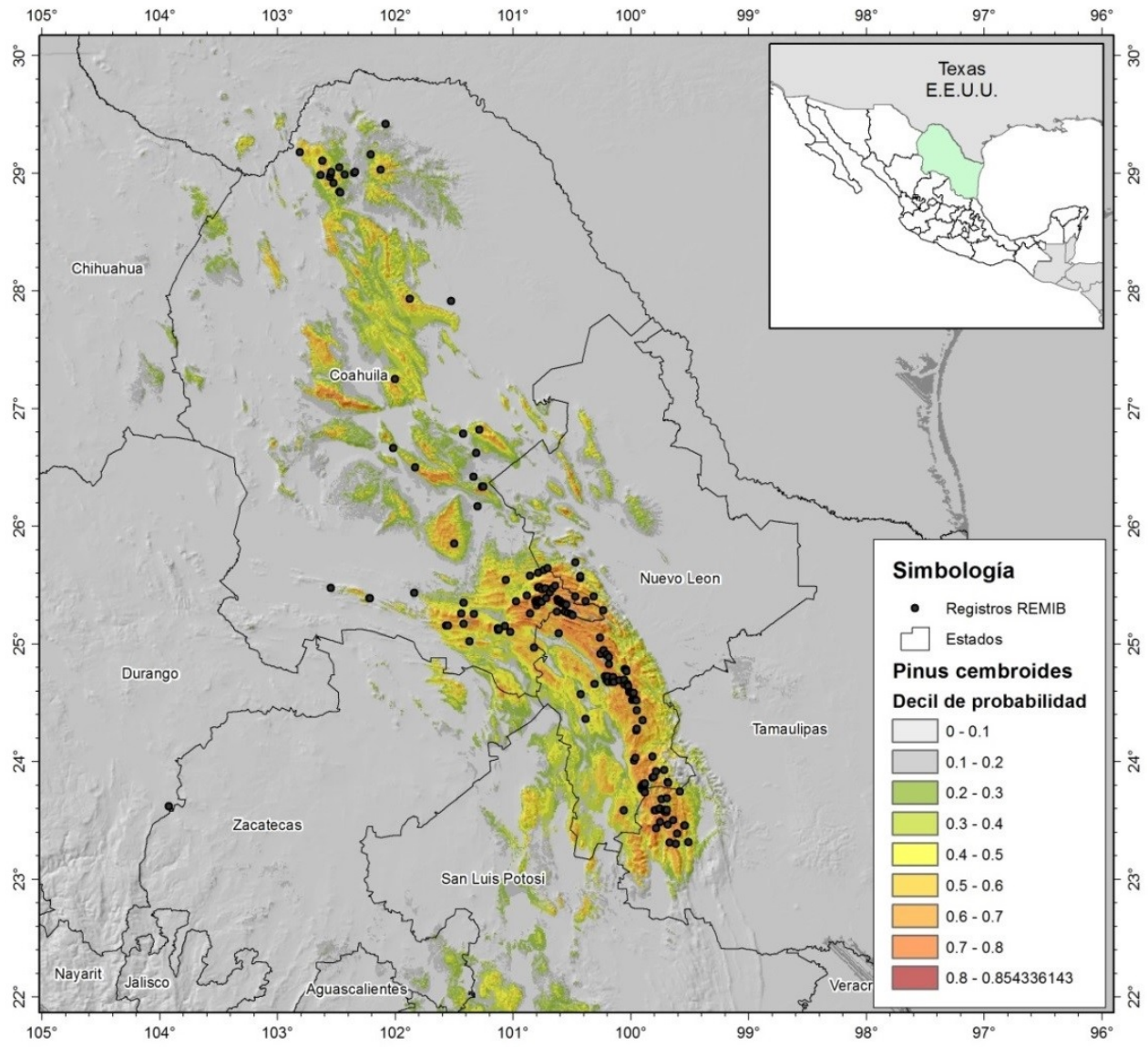

Figura 1. Distribución potencial de P. cembroides en una porción de la SMO, México, basado en variables Bioclim y topográficas. Los últimos dos deciles se consideran como la zona con mejor probabilidad de presencia de la especie.

2 se observa el resultado de la modelación de $P$. nelsonii, donde los valores más cercanos a 1 ( 0.7 - 0.75) se ubican en los límites de Nuevo León y sur de Tamaulipas. Para P. culminicola, el modelo generado muestra que los valores de 0.6 a 0.7 (Tabla 3) se concentran en las zonas de alta montaña entre Coahuila y Nuevo León (Figura 3).

\section{Contribución de las variables en la modelación y mapas de distribución}

Las variables de mayor aportación al modelo generado para $P$. cembroides son la temperatura media del cuarto anual más seco (Bio09), temperatura máxima del mes más cálido (Bio05) y la pendiente con $26.9,11.6$ y $10.1 \%$ respectivamente (Tabla 4), con límites mínimos de 8.2, máximo de 18.7 y media de $12.4{ }^{\circ} \mathrm{C}$ (Figura 4a); 
Tabla 3. Superficie por decil del modelo de hábitat potencial de $P$. cembroides, $P$. nelsonii y $P$. culminicola en el Noreste de México. Los decíles $(0.7-0.85)$ son considerados como la zona de hábitat más probable.

\begin{tabular}{cccccc}
\hline \multicolumn{2}{c}{$\boldsymbol{P}$. cembroides } & \multicolumn{2}{c}{$\boldsymbol{P}$. nelsonii } & \multicolumn{2}{c}{$\boldsymbol{P}$. culminicola } \\
\hline Decil & Superficie $\mathbf{( k m}^{2} \mathbf{)}$ & Decil & Superficie $\mathbf{( k m}^{2} \mathbf{)}$ & Decil & Superficie $\mathbf{( k m}^{2} \mathbf{)}$ \\
\hline $0.0-0.01$ & 214744.12 & $0.0-0.0117$ & 205006.29 & $0.0-0.0055$ & 244311.95 \\
$0.1-0.2$ & 23375.13 & $0.0117-0.1$ & 55838.84 & $0.0055-0.1$ & 41540.18 \\
$0.2-0.3$ & 13402.00 & $0.1-0.2$ & 11031.23 & $0.1-0.2$ & 4691.57 \\
$0.3-0.4$ & 12881.44 & $0.2-0.3$ & 5533.94 & $0.2-0.3$ & 2753.31 \\
$0.4-0.5$ & 10325.95 & $0.3-0.4$ & 5018.98 & $0.3-0.4$ & 1289.22 \\
$0.5-0.6$ & 8946.70 & $0.4-0.5$ & 5886.53 & $0.4-0.5$ & 797.59 \\
$0.6-0.7$ & 8418.35 & $0.5-0.6$ & 5546.82 & $0.5-0.6$ & 485.8 \\
$0.7-0.8$ & 3828.99 & $0.6-0.7$ & 1891.69 & $0.6-0.70$ & 104.32 \\
$0.8-0.85$ & 51.69 & $0.7-0.75$ & 219.66 & - & - \\
\hline
\end{tabular}

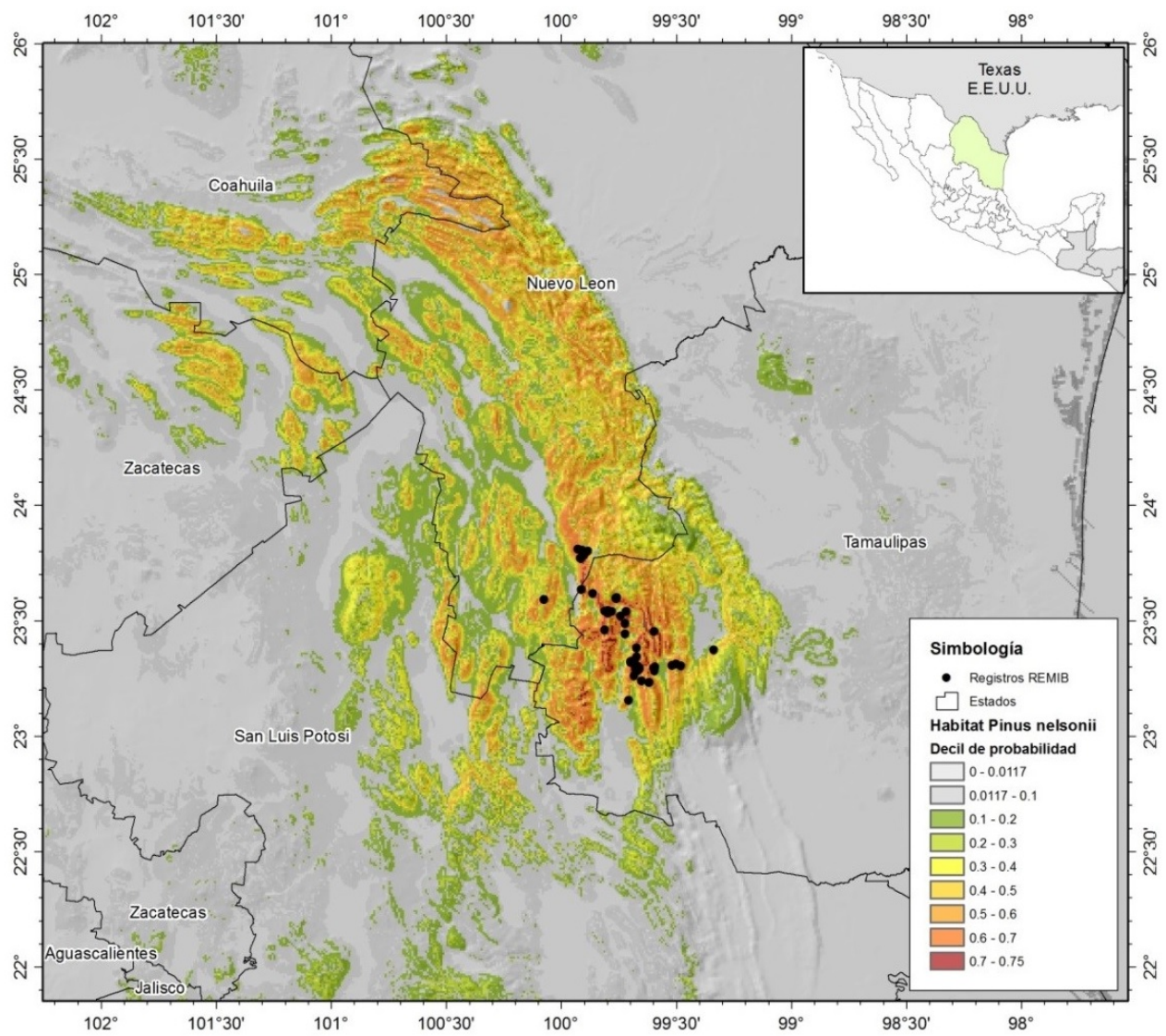

Figura 2. Distribución potencial de P. nelsonii en una porción de la SMO, México, basado en variables Bioclim y topográficas. Los últimos dos deciles se consideran como la zona con mejor probabilidad de presencia de la especie.

mínimo de 21.1 , máximo de 36.7 y media de $28.2{ }^{\circ} \mathrm{C}$ (Figura 4b) y mínimo de 0.3 , máxima de 24.1 y media de 7.4 grados (Figura 4c), respectivamente. La precipitación del cuarto anual más húmedo (Bio16) tuvo $8.8 \%$ de participación, ubicando a $P$. cembroides como una especie con preferencia por lu- gares con lluvias de mediana intensidad en alguna temporada del año, el resto de otras 18 variables no pasan de un $7 \%$ de aportación o menor (Tabla 4).

La altitud y la pendiente fueron variables importantes en la predicción del hábitat de las especies de distribución restringida $(P$. nelsonii y $P$. 


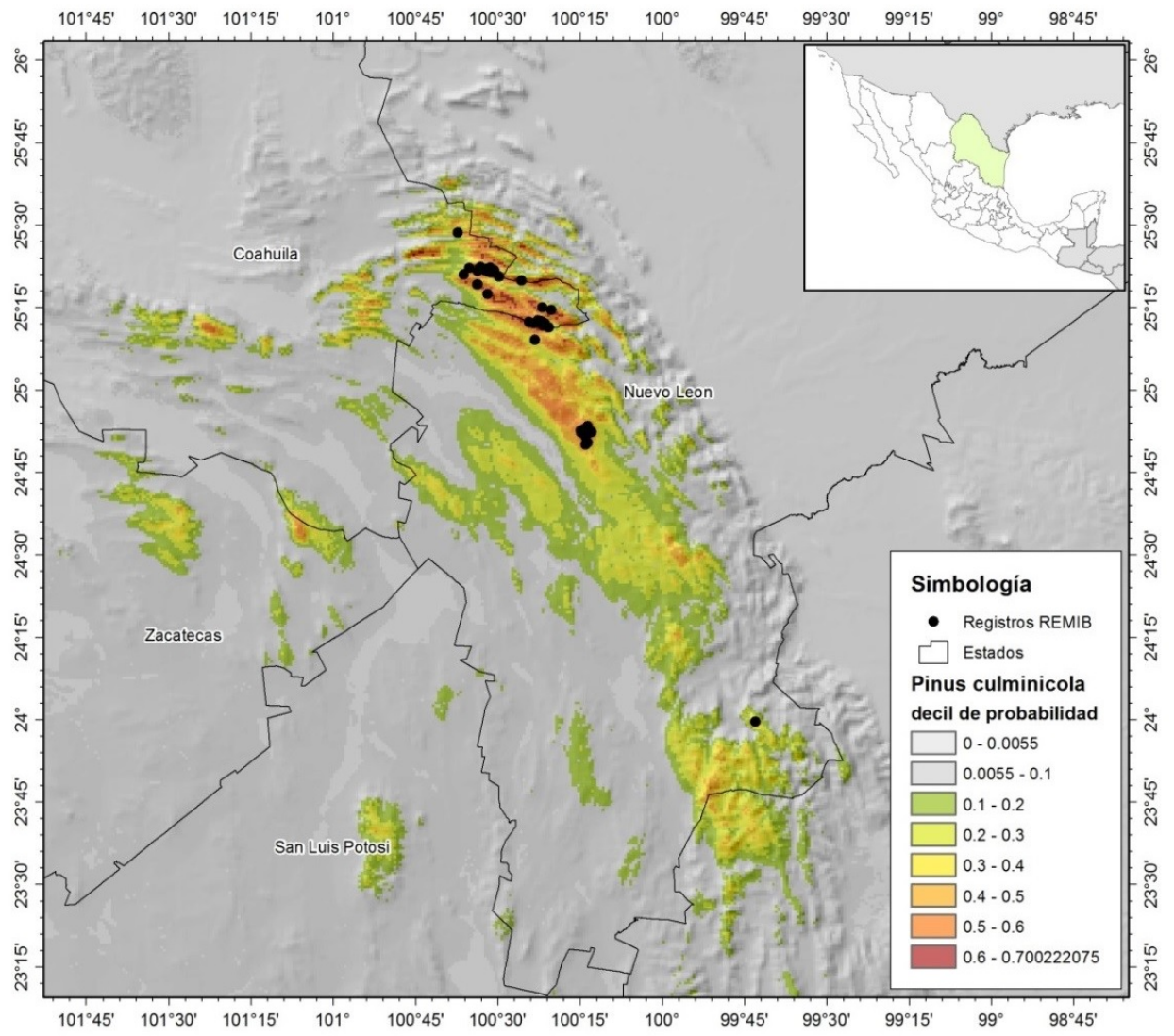

Figura 3. Distribución potencial de $P$. culminicola en una porción de la $\mathrm{SMO}$, México, basado en variables Bioclim y topográficas. Los últimos dos deciles se consideran como la zona con mejor probabilidad de presencia de la especie.

culminicola), aportando al modelo 21.1 y $44.4 \%$ la altitud; 15.0 y $18.3 \%$ la pendiente, para ambas especies, respectivamente. Otra variable que aportó al modelo de predicción de ambas especies fue la temperatura mínima (Tabla 4); para P. nelsonii la temperatura mínima del cuarto anual más frío (Bio11) varió de 2.8 a 7.2 con media de $4.6{ }^{\circ} \mathrm{C}$ (Figura 4d); la altitud fue de 1070 a $2474 \mathrm{~m}$ (Figura 4e) y la pendiente osciló de 2.2 a 15.9 , con media de 7.5 grados (Figura 4f). Para $P$. culminicola la variable temperatura media del cuarto anual más frío varió de 4.7 a $10.5^{\circ} \mathrm{C}$ (Figura $4 \mathrm{~g}$ ), la altitud mínima fue de 2232 y la máxima de 3575 m (Figura 4h), mientras que la pendiente en la que se distribuye la especie es en promedio de 15.0 grados (Figura 4i), el resto de las variables aportan al modelo menos del $8 \%$.
El modelo de MaxEnt predice para P. cembroides una distribución amplia en la Sierra Madre Oriental al sur de Nuevo León, Sureste de Coahuila y posible presencia en las sierras aisladas al centro del estado, y parte de Tamaulipas (Figura 1). De la misma forma, MaxEnt predice la mayor probabilidad (0.70 - 0.75) de ocurrencia de $P$. nelsonii en la zona suroeste de Tamaulipas entre los municipios de Miquihuana, Bustamante, Jaumave, Palmillas y Tula, además de Dr. Arroyo y Zaragoza en Nuevo León, se distribuye en las partes bajas de la sierra de San Antonio, Peña Nevada en zonas de ecotono entre los bosques templados altos y los matorrales áridos colindantes con el altiplano desértico.

La mejor condición potencial, decil de 0.70 para la distribución de $P$. culminicola ocurre zonas 
Tabla 4. Porcentajes de contribución de variables ambientales en la modelación MaxEnt de distribución potencial de $P$. cembroides, $P$. nelsonii y P. culminicola en el Noreste de México.

\begin{tabular}{llccc}
\hline & & $\boldsymbol{P}$. cembroides & $\boldsymbol{P}$. nelsonii & $\boldsymbol{P}$. culminicola \\
\cline { 2 - 5 } Variable & Descripción & 0.7 & - & - \\
\hline Bio01 & Temperatura media anual & 0.9 & - & - \\
Bio02 & Intervalo medio diurno & 0.1 & 0.6 & - \\
Bio03 & Isotermalidad & 3.5 & 9.1 & 4.1 \\
Bio04 & Temporalidad de la temperatura & 11.6 & - & - \\
Bio05 & Temperatura máxima del mes más cálido & 2.1 & 23.7 & - \\
Bio06 & Temperatura mínima del mes más frio & 4.5 & - & - \\
Bio07 & Intervalo de temperatura anual & 26.9 & 0.2 & 0.1 \\
Bio09 & Temperatura media del $\frac{1}{4}$ anual más seco & - & - & 0.1 \\
Bio10 & Temperatura media del $\frac{1}{4}$ anual más cálido & - & 12.9 & 24.4 \\
Bio11 & Temperatura media del $\frac{1}{4}$ anual más frio & 5.5 & 0.2 & 0.4 \\
Bio12 & Precipitación anual & - & - & 0.5 \\
Bio13 & Precipitación del mes más húmedo & 4.1 & 9.7 & 7.8 \\
Bio14 & Precipitación del mes más seco & 7 & - & - \\
Bio15 & Temporalidad de la precipitación & 8.8 & 3.9 & 0.2 \\
Bio16 & Precipitación del $\frac{1}{4}$ anual más húmedo & 6 & 2.3 & - \\
Bio17 & Precipitación del $\frac{1}{4}$ anual más seco & 0.2 & 0.8 & - \\
Bio18 & Precipitación del $\frac{1}{4}$ anual más cálido & 2 & 0.2 & - \\
Bio19 & Precipitación del $\frac{1}{4}$ anual más frio & 5.9 & 21.2 & 44.4 \\
Alt & Altitud & 0.2 & 0.1 & - \\
Exp & Exposición & 10.1 & 15 & 18.3 \\
Pnd & Pendiente & &
\end{tabular}

en Galeana Nuevo León, en el Cerro El Potosí, cima de un grupo de cordilleras de la sierra entre los límites de Coahuila y Nuevo León, como La Viga, La Martha, Potrero Ábrego, El Coahuilón y San Antonio (Figura 3), sitios con una elevación dentro de la distribución conocida de la especie.

\section{Análisis de la superficie de hábitat potencial de las especies estudiadas}

De acuerdo con el modelo generado para $P$. cembroides, la superficie estimada oscila entre 51.6 y $3828.9 \mathrm{~km}^{2}$, lo que representa entre 0.01 y $1.31 \%$ de la superficie de los tres estados del Noreste de México. Para P. nelsonii la superficie potencial es de $219.6 \mathrm{~km}^{2}$. De las tres especies modeladas, $P$. culminicola es la que menos superficie potencial posee con $104.3 \mathrm{~km}^{2}$ (Tabla 3), todo esto considerando superficie estimada a partir del decil 7 (probabilidad $>0.7)$.

\section{DISCUSIÓN}

Considerando el criterio de la cercanía al valor de 1.0 como un mejor modelo (Phillips et al. 2006, Soberón 2011) se supone que el mejor escenario para predecir la distribución potencial de $P$. cembroides es aquel que incluye las 19 variables Bioclim más las variables topográficas, con valores AUC mayores en el set de datos y en el de entrenamiento, donde se establece la influencia de la topografía en la modelación de la distribución potencial de éstas especies (García et al. 2012). Para especies de distribución restringida ( $P$. nelsonii y $P$. culminicola) ambos escenarios predicen de forma adecuada la distribución potencial, por lo que puede usarse cualquiera de ellos. Estudiando estas mismas especies pero con otro fin, Aguirre y Duivenvoorden (2010) obtuvieron valores AUC de 0.895, 0.933 y 0.990 para $P$. cembroides var. cembroides, $P$. nelsonii y $P$. culminicola respectivamente, valores altos que variaron con los del presente estudio por utilizar distintas cubiertas ambientales, de menor resolución además de haber usado una fuente distinta de registros de presencia (BRAHMS). Las principales variables predictoras para $P$. cembroides (Bio09 y Bio05) indican una adaptación de la especie a ambientes de temperaturas templadas con veranos cálidos, característica de bosques de piñonero de las zonas de altiplano (Perry 1991, Farjon y Styles 1997) y se asocia hasta con ocho comunidades 

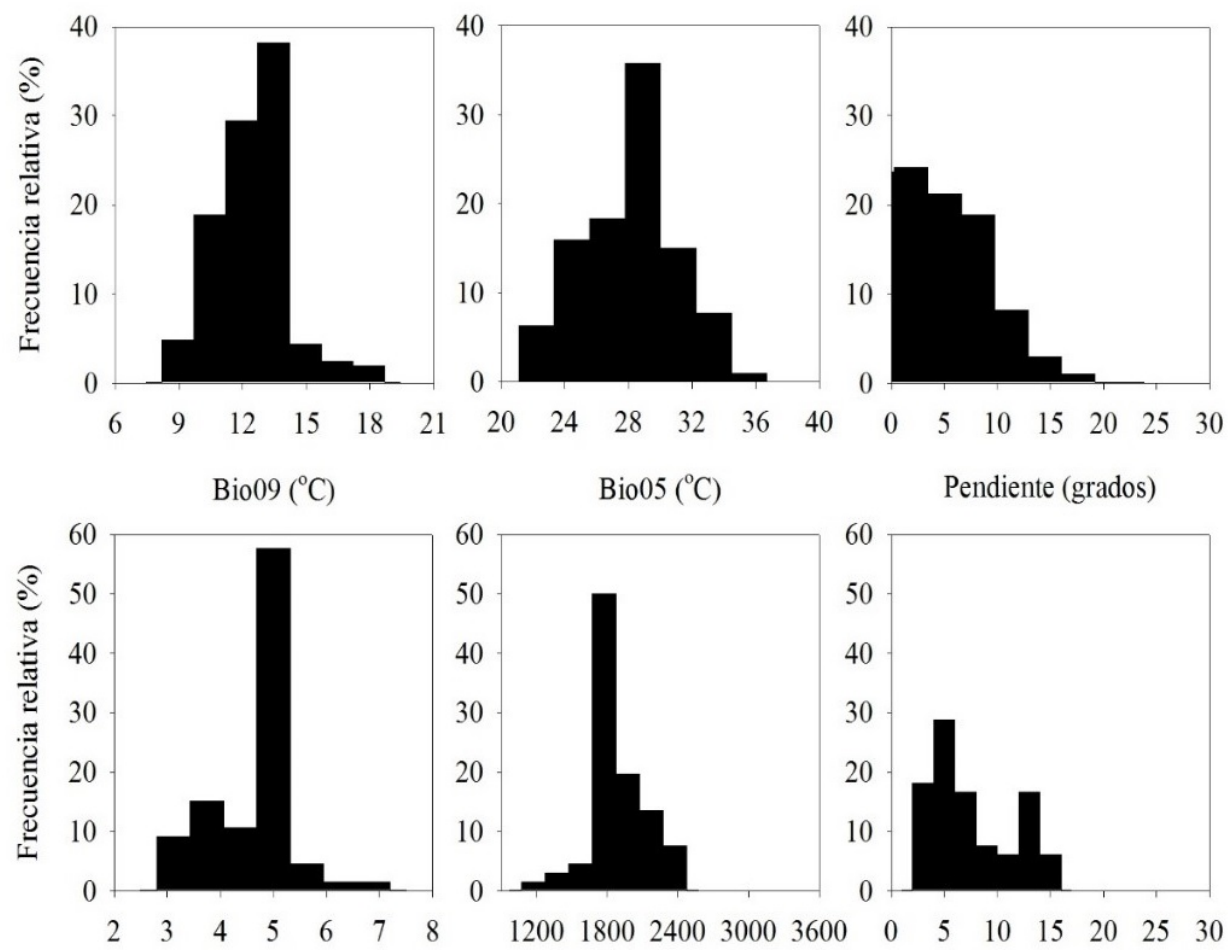

$\operatorname{Bio05}\left({ }^{\circ} \mathrm{C}\right)$

Pendiente (grados)
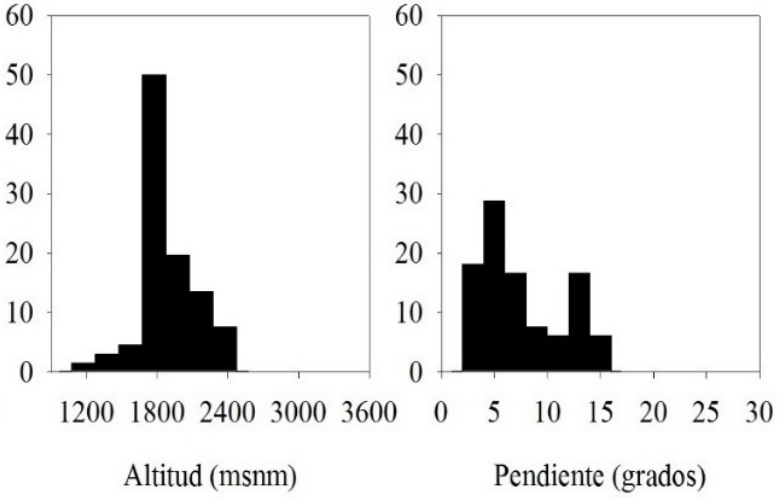

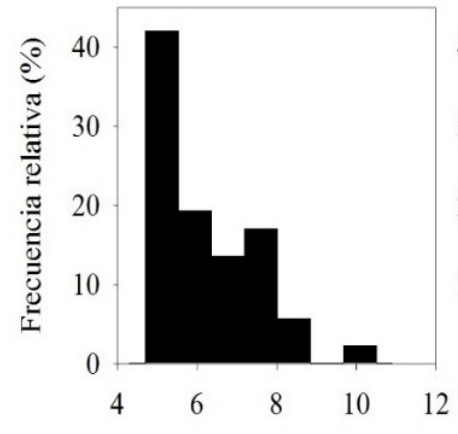

Bioll $\left({ }^{\circ} \mathrm{C}\right)$
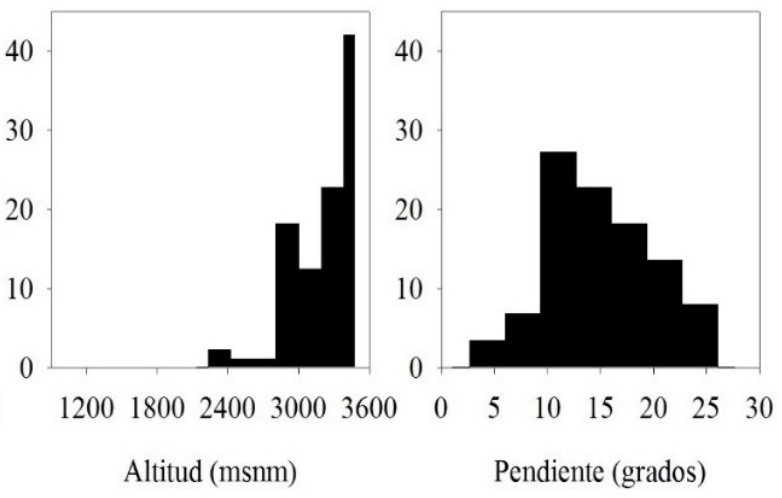

Figura 4. Histogramas de frecuencia de tres variables de mayor influencia en los modelos de distribución potencial de: Pinus cembroides $(\mathrm{a}-\mathrm{c}$ ), Pinus nelsonii $(\mathrm{d}-\mathrm{f})$ y Pinus culminicola $(\mathrm{g}$ - i) en una porción de la SMO, México.

vegetales (Estrada et al. 2014). Al respecto Passini (1982) reporta que la distribución de $P$. cembroides en la Sierra Madre Oriental se encuentra entre 250 y $400 \mathrm{~mm}$ de precipitación anual y de 17 a $20{ }^{\circ} \mathrm{C}$ de temperatura. El modelo generado en este estudio al igual que el de Aguirre y Duivenvoorden (2010) corrobora la amplia distribución potencial de $P$. cembroides, y reportada por otros autores (Estrada et al. 2014).

La especie $P$. nelsonii se distribuye entre los 1600 y $2300 \mathrm{msnm}$, en zonas con precipitación anual entre 300 y $600 \mathrm{~mm}$, y se ubica en pies de 
montaña de ambientes áridos a semiáridos (Perry 1991, Farjon y Styles 1997). Mientras que Estrada et al. (2014), reportan que distribuye en ecotonos entre matorrales, chaparrales de encino y bosques de $P$. cembroides. En lo referente a $P$. culminicola se reporta que se distribuye de los 3000 a los 3 $700 \mathrm{msnm}$, bajo condiciones de vientos fuertes, exposición preferentemente norte, creciendo a bajas temperaturas (Perry 1991, Farjon y Styles 1997), es una de las especies más vulnerables a la reducción de su hábitat por efecto del cambio climático (Gómez y Arriaga 2007). Lo resultados encontrados junto a lo reportado por Aguirre y Duivenvoorden (2010), refuerzan la observación de que $P$. nelsonii y $P$. culminicola presentan una distribución restringida, aunque existen regiones modeladas donde no se tiene registro, donde MaxEnt predice hábitat potencial que puede ser considerado para promover actividades de conservación, ya que son especies en estatus de protección y amenazadas.

Las variables que mejor predicen la distribución potencial del género Pinus, sobre todo de especies de distribución amplia se relacionan con las temperaturas altas (Cruz et al. 2012), para especies que poseen distribución restringida, la altitud predomina sobre temperatura, no obstante esto parece ser específico, ya que para especies de Abies la altitud y exposición predominan sobre las temperaturas (Martínez et al. 2016). Las variaciones en el ajuste del modelo y de la distribución potencial de las especies depende del tipo de variables bioclimáticas, escala y número de registros de especies (Aguirre y Duivenvoorden 2010, Martínez et al. 2016).

La superficie calculada de $P$. culminicola, se considera sobreestimada debido a que en el pasado, incendios forestales mermaron de forma significativa la superficie original. Por ejemplo, en 1998 la superficie de $P$. culminicola en el Cerro El Potosí se redujo un $40 \%$, reportándose únicamente 30 ha (Estrada et al. 2014), por lo que las 10400 ha calculadas con el modelo deben interpretarse como zonas con el bioclima y la topografía óptimas para su posible distribución o futuros estudios de repoblación.

\section{CONCLUSIONES}

Las variables Bioclim y topográficas modelan de forma adecuada la distribución potencial de $P$. cembroides, P. culminicola y $P$. nelsonii; siendo las que mejor definen el hábitat potencial la temperatura máxima y media de los meses más secos, para $P$. cembroides la temperatura mínima del mes más frío, la altitud en $P$. nelsonii, y para $P$. culminicola la altitud y la temperatura media del más frío. Para las tres especies, la pendiente determina su distribución potencial. El modelo predice amplia distribución potencial de $P$. cembroides, que puede incrementarse en el futuro debido a que el modelo integró variables dependientes de temperatura máxima y discriminó aquellas de derivadas de precipitación. Las áreas de mayor probabilidad de ocurrencia de $P$. nelsonii y $P$. culminicola dependien de la altitud, la pendiente y las temperaturas frías.

\section{AGRADECIMIENTOS}

Al CONACyT por la beca otorgada al tercer autor para realizar sus estudios de posgrado en la Universidad Autónoma Agraria Antonio Narro.

\section{LITERATURA CITADA}

Aguirre GJ, Duivenvoorden JF (2010) ¿Can we expect to protect threatened species in protected areas? A case study of the genus Pinus in Mexico? Revista Mexicana de Biodiversidad 81: 875-882.

Austin MP, Meyers JA (1996) Current approaches to modelling the environmental niche of eucalypts: implication for management of forest biodiversity. Forest Ecology Management 85: 95-106.

Austin MP, Smith TM (1989) A new model for the continuum concept. Vegetatio 83: 35-47.

Ávila CR, Villavicencio GR, Ruiz CJA (2014) Distribución potencial de Pinus herrerae Martínez en el occidente del estado de Jalisco. Revista Mexicana de Ciencias Forestales 5: 92-109. 
CONABIO (2015) REMIB Red mundial de información sobre biodiversidad. Comisión nacional para el conocimiento y uso de la biodiversidad. http://www.conabio.gob.mx. Fecha de consulta 20 de noviembre de 2016.

Cruz CG, Villaseñor JL, López ML, Ortiz E (2012) Potential distribution of humid mountain forest In México. Botanical Sciences 9: 331-340.

De la Torre JA, Torres KI (2014) Distribución potencial del puma (Puma concolor) en el estado de Aguascalientes, México. Revista Mexicana de Mastozoología 4: 45-56.

Estrada CE, Villarreal J, Salinas M, Encina J, Cantú C, González H, et al. (2014) Coníferas de Nuevo León, México. Facultad de Ciencias Forestales, Universidad Autónoma de Nuevo León Nuevo León, México. $144 p$.

Farjon A, Styles B (1997) Pinus (Pinaceae). Flora Neotropica, Monograph 75. The New York Botanical Garden. Bronx, New York. 291p.

García AM, Cantú AC, Estrada CE, Pando MM, Moreno TA (2012) Distribución actual y potencial de Taxus globosa (Taxaceae) en México. Journal of the Botanical Research Institute of Texas 6: 587-598.

Gómez ML, Arriaga L (2007) Modeling the effect of climate change on the distribution of oak and pine species of Mexico. Conservation Biology 21: 1545-1555.

Graham CH, Ferrier S, Huettman F, Moritz C, Peterson AT (2004) New developments in museum-based informatics and applications in biodiversity analysis. Trends in Ecology \& Evolution 19: 497-503.

Hijmans RJ, Cameron SE, Parra JL, Jones PG, Jarvis A (2005) Very high resolution interpolated climate surfaces for global land areas. International Journal of Climatology 25: 1965-1978.

Kumar S, Stohlgren TJ (2009) MaxEnt modeling for predicting suitable habitat for threatened and endangered tree Canacomyrica monticola in New Caledonia. Journal of Ecology and Natural Environment 1: 094098.

Lawler JJ, Shafer SL, White D, Kareiva P, Maurer EP, Blaustein AR, et al. (2009) Projected climate-induced faunal change in the western hemisphere. Ecology 90: 588-597.

Martínez MN, Aguirre PE, Eguiarte LE, Jaramillo CJ (2016) Modelado de nicho ecológico de las especies del género Abies (Pinaceae) en México: Algunas implicaciones taxonómicas y para la conservación. Botanical Sciences 94: 5-24.

Martínez N (2010) Apuntes sobre modelación de nichos ecológicos. Laboratorio de evolución molecular y experimental, del Instituto de Ecología de la Universidad Nacional Autónoma de México. https://www.yum pu.com/es/document/view/14874920/2010-apuntes-sobre-modelacion- de-nichos-ecologicos-institutode-/3. Fecha de consulta 3 de junio de 2016.

Meentemeyer RK, Anacker BL, Mark W, Rizzo DM (2008) Early detection of emerging forest disease using dispersal estimation and ecological niche modeling. Ecological Applications 2: 377-90.

Nix HA (1986) BIOCLIM - A bioclimatic analysis and prediction system. CSIRO Division of Water and Land Resources Research (Ed.). Annual Report. CSIRO Publishing. Canberra, Australia. pp: 59-60.

Passini MF (1982) Les Forêts de Pinus cembroides au Mexique. Mission Arquèologique et ethnologique Francaise au Mexique. Etudes Mèsoamèricaines II-5, Edit. Rech. Sur les civilizations. Paris. 373p.

Perry J (1991) The pines of Mexico and Central America. Timber Press. Portland Oregon, USA. 231p. 
Phillips SJ, Anderson RP, Schapire RE (2006) Maximum entropy modeling of species geographic distributions. Ecological Modelling 190: 231-259.

Salinas-Moreno Y, Ager A, Vargas C, Hayes J, Zuñiga G (2010) Determining the vulnerability of Mexican pine forests to bark beetles of the genus Dendroctonus Erichson (Coleoptera: Curculionidae: Scolytinae). Forest Ecology and Management 260: 52-61.

Shaily M, Baharul I, Choudhury M, Latif K, Townsend PA (2010) Ecological niche modeling and local knowledge predict new populations of Gymnocladus assamicus a critically endangered tree species. Endangered Species Research 11: 175-181.

Soberón J (2011) Nichos y áreas de distribución, Validación del modelo, CONABIO-University of Kansas. Comisión Nacional de Biodiversidad, México. http://nicho.conabio.gob.mx/home/proposito-y-guia-delusuario/validacion-del-modelo. Fecha de consulta 2 de marzo de 2017.

Stockwell D (1999) The GARP modelling system: problems and solutions to automated spatial prediction. International Journal of Geographical Information Science 13: 143-158.

Valdés OT, Huerta YMC, Chávez AGT, Garduño MVG (2012) Modelado bioclimático como herramienta para el manejo forestal: Estudio de cuatro especies de Pinus. Revista Mexicana de Ciencias Forestales 29: $1-22$. 
